\title{
Comércio Eletrônico: Uma Análise da Aplicabilidade de Modelos de Qualidade de Software
}

\author{
Luiz Alberto Ferreira Gomes \\ Mario Lúcio Côrtes \\ Universidade Estadual de Campinas - UNICAMP \\ Instituto de Computação - IC \\ Avenida Albert Einstein, 1251. \\ Caixa Postal, 6176. \\ 13083.970, Campinas, SP. \\ \{luiz.gomes, cortes\}@ic.unicamp.br
}

\begin{abstract}
Resumo
O rápido desenvolvimento do comércio eletrônico está provocando a evolução das técnicas de desenvolvimento de software. Formalmente, se conhece muito pouco sobre os impactos deste movimento sobre a qualidade de software. Este artigo apresenta os resultados preliminares de uma pesquisa cujo objetivo é analisar a aplicabilidade de modelos de qualidade de software no desenvolvimento de aplicações para comércio eletrônico. A análise é baseada na percepção de vários autores na literatura e de dados coletados em entrevistas com gerentes de projeto de organizações brasileiras de comércio eletrônico.
\end{abstract}

Palavras-chave: Comércio Eletrônico, Qualidade de Software, CMM, CMMI, e $\mathrm{C}^{\mathrm{CCM}}$.

\begin{abstract}
The rapid development of e-Commerce is posing new demands on software development techniques. The impact of this on software quality is not well understood. This paper presents preliminary findings of a research, which aims at analyzing the applicability of quality software models to e-Commerce application development. The analysis is based on the perception of several authors in the literature and from data gathered in interviews with project managers of the Brazilian e-Commerce organizations.
\end{abstract}

Keywords: Electronic Commerce, Software Quality, CMM, CMMI, e ${ }^{\mathrm{CCM}}$.

\section{Introdução}

O uso cada vez maior da Internet pelas empresas está transformando os processos de negócio por meio: da diminuição das barreiras entre clientes e fornecedores; da melhoria dos serviços prestados; da redução dos ciclos de produção; e da introdução de novas formas de comercialização eletrônica de produtos [9]. O comércio eletrônico, descrito brevemente na seção 2, dá mostras de um potencial muito grande de mercado, mesmo com as dificuldades de várias empresas "dotcom" [3]. Nota-se, também, que o comércio eletrônico está influenciando positivamente os números da economia brasileira com negócios da ordem de 50 bilhões de reais[15].

O comércio eletrônico, além de ser apoiado em uma estratégia de negócios bem definida, é fortemente baseado em aplicações de software para o ambiente Web. Apesar 
dessas serem aplicações como outras quaisquer, existem diferenças que criam dificuldades particulares no processo de desenvolvimento de software em relação ao ambiente convencional. Ciclos de desenvolvimento extremamente reduzidos, intensas alterações de requisitos, freqüentes mudanças tecnológicas e a falta de profissionais experientes são alguns dos problemas ou aspectos do desenvolvimento para ambiente Web[14]. Claramente, existe a necessidade de um modelo de desenvolvimento de software ágil, adaptável e que traga embutido um caminho que oriente as empresas de comércio eletrônico a atingir um razoável nível de qualidade no desenvolvimento de aplicações.

Os modelos apresentados nas seções 3 e 4, o Capability Maturity Model (CMM) e o CMM-Integrated (CMMI) respectivamente, são referências quando se fala em modelos de qualidade de software. Possuem boa aceitabilidade, obtiveram um expressivo sucesso no desenvolvimento de aplicações convencionais e são amplamente difundidos na indústria de software. Contudo, eles estão sofrendo fortes críticas quando se trata da sua aplicabilidade no desenvolvimento de aplicações para comércio eletrônico [18]. O Software Engineering Institute (SEI) em parceria com a companhia norte-americana Boeing Company está desenvolvendo um modelo específico de desenvolvimento de comércio eletrônico, chamado eCommerce Capability Model $\left(\mathrm{e}^{\mathrm{CCM}}\right)$. Não se tem ainda um documento formal sobre o modelo, mas algumas informações preliminares a seu respeito são apresentados na seção 5.

Mesmo com o rápido desenvolvimento do comércio eletrônico, segundo [6], formalmente se conhece muito pouco de que maneira as técnicas de desenvolvimento de software empregadas pelas empresas afetam a qualidade de software. Este artigo tem por objetivo mostrar os resultados preliminares de um projeto de pesquisa, ainda em andamento, que analisará o grau de aplicabilidade dos modelos existentes CMM, CMMI e novo e ${ }^{\mathrm{CCM}}$ no desenvolvimento de aplicações para comércio eletrônico. Eventualmente, os resultados encontrados possibilitarão a sugestão de propostas de melhorias que possam adequar os modelos estudados à nova realidade. O projeto segue a metodologia apresentada na seção 6 . A seção 7 apresenta os resultados preliminares, a seção 8 mostra as conclusões e a seção 9 as referências utilizadas neste artigo.

\section{Comércio Eletrônico}

Comércio eletrônico pode ser definido como "a compra e venda de informações, produtos e serviços, além de todos os serviços de pré e pós-venda realizados através de computadores incluindo-se nesse segmento a Internet e todas as mídias interativas englobando vários processos de negócios da organização" [4]. Dependendo das partes envolvidas (vendedor ou comprador), o comércio eletrônico pode ser classificado em "business-toconsumer" (B2C) ou "business-to-business" (B2B). No B2C a negociação é realizada entre uma empresa e um consumidor diretamente e no B2B a negociação ocorre entre duas empresas.

O comércio eletrônico viabilizou a criação de mercados globais através da eliminação das distâncias entre fornecedor e consumidor. Criou-se uma nova geografia virtual em que só existe uma economia e um mercado com potencial muito maior que os mercados atuais. Outros benefícios que demonstram a importância do comércio eletrônico para empresas, pessoas ou organizações governamentais são os seguintes [4]:

- Compra direta, ou seja, eliminação do atravessador;

- Redução de custos; 
- Situação de comando por parte do comprador;

- Inexistência de horários de funcionamento para transações comerciais;

- Entrega eletrônica para alguns tipos de produtos e serviços.

Como se pode ver, os benefícios são muitos. Contudo, o comércio eletrônico por estar inserido em um ambiente extremamente dinâmico e dirigido fortemente por pressões de mercado, deixa quaisquer implementações de software sujeitas a problemas que o desenvolvimento de aplicações convencionais não sofria. O CMM, brevemente mostrado na próxima seção, é um modelo que ajudou a melhorar a qualidade dos processos de desenvolvimento e a resolver diversos problemas de qualidade de software convencional, mas que ainda é pouco utilizado ou chama pouco a atenção de empresas de comércio eletrônico[5].

\section{CMM}

Para tentar resolver inúmeros problemas de desenvolvimento de projetos de software que enfrentava, o Departamento de Defesa norte-americano (DoD) patrocinou a criação do SEI, que desde o início foi gerenciado pela Carnegie Mellon University (CMU). O SEI foi fundado com a responsabilidade de criar ou avançar o conhecimento na área de Engenharia de Software. Uma de suas notáveis criações, que se tornou bastante difundida não só no âmbito do DoD mas também em toda indústria de software, foi o CMM. Para informações e detalhes adicionais sobre o modelo, consultar as referências [11] e [1].

O CMM é um dos modelos de qualidade de software mais consolidados da área de software e aplicável, segundo o próprio SEI, em projetos de software de qualquer natureza e em quaisquer ambientes [10]. Entretanto, alguns autores, como Yourdon [18], advogam que o CMM é muito pesado e burocrático para atender às necessidades e se adaptar às características do ambiente Web. Em outro artigo [17], o autor pondera que embora ele possuísse um conhecimento formal sobre o modelo CMM, o modelo não foi aplicado diretamente no processo de concepção e implantação de um processo de melhoria de desenvolvimento de aplicações de comércio eletrônico da empresa Eastman Kodak. Apenas algumas idéias inspiraram-se nele. A justificativa do autor é que a empresa não estava buscando alcançar um determinado nível do CMM como objetivo principal.

O autor Mark Paulk[10], rebate estas críticas ou ponderações a respeito do modelo argumentando que para se atingir o nível máximo do CMM, são necessárias somente a satisfação de 52 metas e 18 áreas-chave de processos, que as práticas e as sub-práticas são materiais informativos e que o bom senso e o conhecimento profissional de quem está implementando deverão orientar o gerenciamento dos processos.

Na próxima seção, será visto a nova versão do modelo CMM que incorpora várias melhorias sugeridas por aqueles que o implementaram. Esta nova versão é conhecida como CMMI.

\section{CMMI}

O SEI, desde 1991, tem emitido documentos derivados do CMM para uma diversidade de setores da indústria de software. Alguns dos mais expressivos incluem os modelos de Engenharia de Sistemas (SE-CMM), aquisição de software (SA-CMM) e gestão de recursos humanos em empresas de software (P-CMM). Embora estes modelos tenham utilidade comprovada na prática, em muitas organizações a utilização de múltiplos modelos trouxe 
inúmeros problemas de integração. Com intuito de unificar estes e outros modelos baseados no CMM, o SEI desenvolveu o CMMI.

\subsection{Modalidades}

Talvez a grande novidade do CMMI seja o oferecimento de duas modalidades de representação: modalidade contínua e modalidade escalonada (staged). As informações elementares contidas nas duas representações são virtualmente as mesmas. Entretanto, cada representação pode ser utilizada de forma diferente pelas organizações [12].

$\mathrm{Na}$ representação contínua, o foco ou componentes principais são as áreas de processo. Nesta representação, há metas e práticas de dois tipos: específicas a uma determinada área de processos e genéricas aplicáveis indistintamente a todas as áreas de processos. A partir da avaliação e do atendimento dessas práticas e metas é possível classificar o nível de maturidade de cada área de processo, em níveis de zero a cinco.

Já na representação escalonada, os componentes principais são níveis de maturidade. Dentro de cada nível, existem áreas de processos que contêm metas, características comuns e práticas. $\mathrm{Na}$ representação em níveis, as práticas são caracterizadas pelos atributos: compromisso para execução (práticas que garantem que o processo seja estabelecido e apoiado); habilidade para execução (práticas que criam condições para que o processo seja estabelecido completamente) e atividade para execução (práticas que implementam diretamente o processo); controle e verificação de implementação.

A representação contínua consiste de seis (0 a 5) níveis de capabilidade que se aplicam na melhoria de áreas de processos individuais, enquanto a representação escalonada consiste de cinco ( 1 a 5) níveis de maturidade que são aplicados na melhoria de processos que abrangem toda a organização. Se uma organização deseja focar seus esforços de melhoria em áreas pré-definidas ela deverá optar pela representação contínua. Entretanto, se a organização deseja um caminho definido para melhoria de processo e que possibilite uma forma de comparação com outras organizações, o caminho a ser seguido é a representação escalonada. Para informações e detalhes adicionais sobre o modelo, consultar as referências [13] e [1].

A adoção do CMMI ainda não atingiu a amplitude do seu antecessor, mas mesmo com algumas áreas-chave, metas e práticas novas, ele possui as mesmas limitações que CMM quanto ao desenvolvimento de aplicações para comércio eletrônico [7]. O modelo descrito em seguida, o $\mathrm{e}^{\mathrm{CCM}}$, é um modelo de desenvolvimento de comércio eletrônico que, aparentemente, traz embutido em si respostas a questões de qualidade de software. Um fator limitante com relação a esse modelo é que ainda não foram divulgados documentos formais sobre ele, nem tampouco resultados práticos quanto a sua implementação.

\section{5. eCommerce Capability Model (e $\left.\mathrm{e}^{\mathrm{CCM}}\right)$}

Resultado de um esforço conjunto da Carnegie Mellon University e da companhia norte-americana Boeing, o $\mathrm{e}^{\mathrm{CCM}}$ surgiu da necessidade do mercado de um modelo de desenvolvimento de comércio eletrônico. Os seus autores tinham em mente criar um modelo que tratasse tanto de questões técnicas quanto de questões de negócios de forma integrada. Ele pode ser definido como uma abordagem sistemática para a implementação de comércio eletrônico, com os objetivos de [16]:

1. Identificar fatores chave associados ao comércio eletrônico; 
2. Identificar habilidade, conhecimento e infra-estrutura tecnológica necessários para implementação de comércio eletrônico;

3. Priorizar ações baseadas em áreas de interesse que podem estar no contexto de unidade de projetos, unidade de negócios, empresa e relacionamento entre empresas;

4. Gerenciar mudanças sistematicamente de maneira veloz, adaptável e com qualidade.

\subsection{Níveis}

O e $\mathrm{e}^{\mathrm{CCM}}$, assim como o CMM, é dividido em níveis de capabilidade. Cada nível consiste de várias áreas-chave e essas áreas são coleções de práticas, abrangendo negócios e tecnologia, que devem ser implementadas para atingir os resultados desejados. A Figura 1 mostra os quatro níveis de capabilidade do modelo.

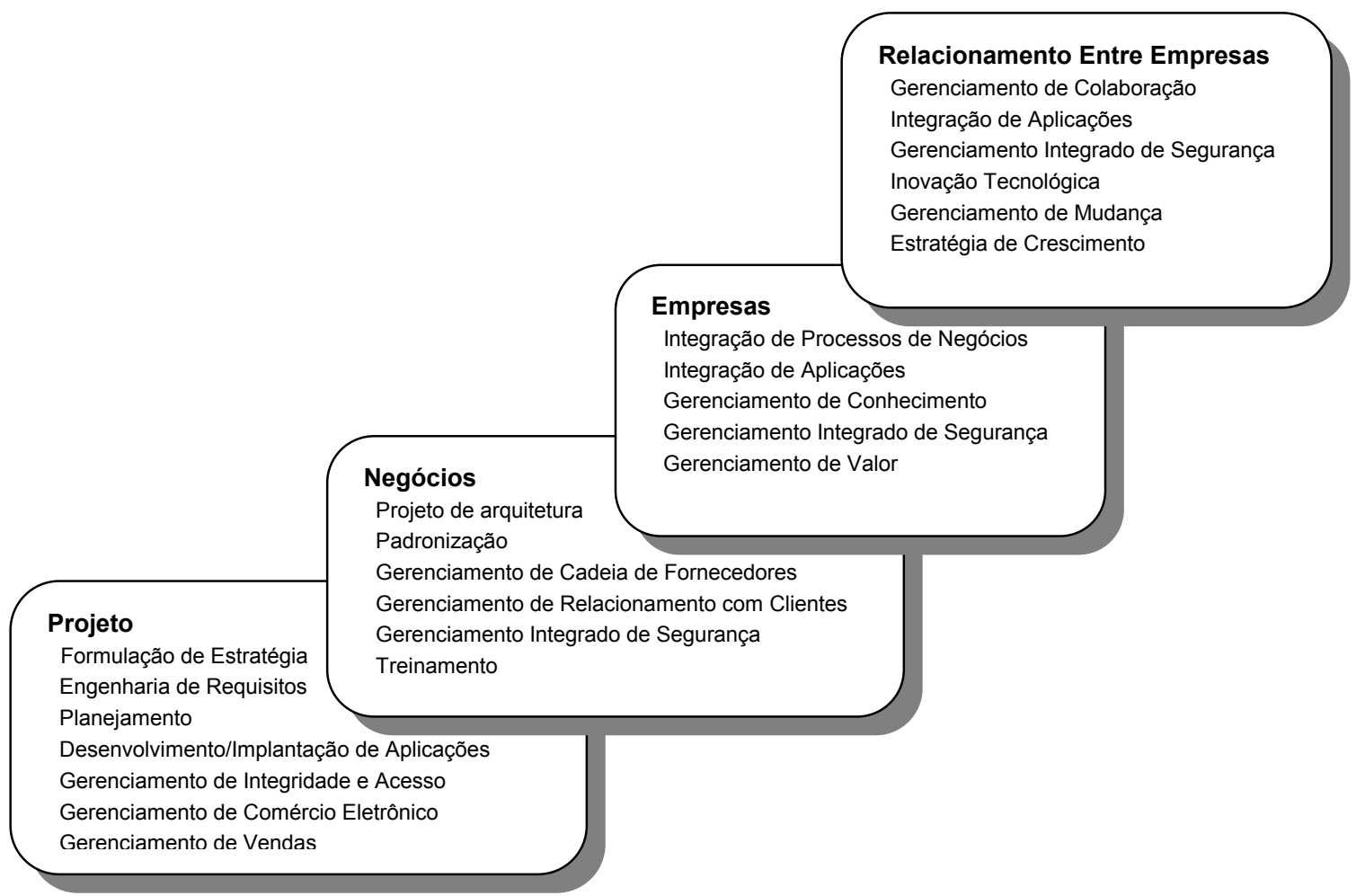

Figura 1: Níveis e áreas-chave do $\mathrm{e}^{\mathrm{CCM}}$.

O primeiro nível, capabilidade de unidade de Projeto, tem por objetivo desenvolver a capacidade para implantar projetos de comércio eletrônico dentro de uma unidade de negócios com velocidade e segurança. O segundo nível, capabilidade de unidade de Negócios, tem por objetivo desenvolver a capacidade para integrar e padronizar projetos de negócios de maneira consistente. O terceiro nível, capabilidade de Empresa, tem por objetivo desenvolver a capacidade de gerenciar todas as unidades de negócios e integrá-los de forma coesa para alcançar o máximo de benefícios. O último nível, Relacionamento entre Empresas, tem por 
objetivo desenvolver a capacidade de integrar processos de negócios que se complementam ou são comuns entre empresas que possuem algum tipo de parceria e elaborar e implementar estratégias para expandir o comércio eletrônico em direção a novos mercados.

Para analisar ou investigar de forma sistemática e organizada a aplicabilidade destes modelos no desenvolvimento de aplicações para comércio eletrônico foi adotada a metodologia descrita na próxima seção. $\mathrm{O}$ projeto se encontra no final da primeira das três etapas previstas na metodologia.

\section{Metodologia do Projeto de Pesquisa}

A metodologia traçada para atingir os objetivos propostos pelo projeto compreende as seguintes etapas:

1. Levantamento de problemas: As principais fontes para levantamento de problemas serão publicações da literatura (livros, artigos, apresentações) e e-mails trocados com os autores das obras. Além disso, com o objetivo de complementar as fontes primárias serão realizadas algumas entrevistas com gerentes de projetos de empresas brasileiras que desenvolveram aplicações para comércio eletrônico.

2. Análise de problemas e modelos: Objetiva determinar qual área-chave, se aplicada, provê uma solução para determinado problema e se a solução é completa ou parcial, ou seja, se as práticas são suficientes ou não; verificar a existência de práticas pouco relacionadas a problemas práticos e relacionar os problemas que não são tratados por nenhuma área-chave.

3. Propostas de melhorias: Tem por objetivo, com base nas duas etapas anteriores, identificar deficiências e propor novas interpretações e orientações para a implantação dos modelos. Além disso, um resultado colateral pode ser a proposta de melhoria nos modelos em desenvolvimento, quanto à inserção, deslocamento, remoção ou mesmo nova interpretação de metas, práticas, sub-práticas e atividades contidas em cada elemento de modelos.

Os resultados já alcançados com a primeira etapa, de levantamento de problemas, são mostrados na seção seguinte.

\section{Resultados Preliminares}

Antes de apresentar os problemas propriamente ditos, a seção 7.1 mostra os fatores que levaram ao surgimento e as características de um novo processo de desenvolvimento de software para comércio eletrônico. Nesse processo, identificado em [5], muitos fatores relacionados à qualidade de software não são considerados como críticos. Todavia, é nesse mesmo contexto que os modelos estudados deveriam atuar em processos de melhoria de desenvolvimento de software nas empresas de comércio eletrônico.

Os problemas levantados foram divididos em três categorias distintas: problemas gerais encontrados na literatura que resultaram da percepção que alguns autores tem do assunto (seção 7.2); problemas levantados junto a empresas brasileiras que desenvolvem aplicações para comércio eletrônico (seção 7.3); e problemas específicos encontrados na literatura, onde constam dados levantados dentro de determinadas empresas (seção 7.4). Mesmo com um número ainda pequeno de empresas entrevistadas, essas informações constituem uma visão abrangente sobre os problemas existentes na área e oferecem uma base 
consistente para a próxima etapa do projeto. A seção 7.5 apresenta uma análise preliminar dos problemas.

\subsection{Características.}

Em um levantamento realizado em vinte empresas dos Estados Unidos e da Dinamarca entre o verão de 2000 e a primavera de 2002, observaram-se os seguintes fatores em empresas que implementaram comércio eletrônico[5]:

- Tipo de mercado diferente dos existentes;

- Falta de experiência dos profissionais envolvidos;

- Extrema pressão de tempo para chegar ao mercado;

- Qualidade do software pode ser negociada;

- Mudança cultural.

Esses fatores estão resultando em um novo processo de desenvolvimento de software com as seguintes características:

- Orientação a versões;

- Desenvolvimento paralelo;

- Manutenção não considerada;

- Prototipação;

- Foco na arquitetura;

- Dependência de ferramentas;

- Forte envolvimento do cliente nas equipes de desenvolvimento;

- Metodologias personalizadas (Tailored Methodology);

- Desenvolvimento baseado em componentes.

\subsection{Problemas Gerais}

Na categoria de problemas gerais, os problemas extraídos de [16] e [14] são mostrados na tabela abaixo:

Tabela 1: Descrição de problemas gerais encontrados na literatura.

\begin{tabular}{|l|l|}
\hline \multicolumn{1}{|c|}{ Vu [16] } & \multicolumn{1}{c|}{ Strigel [14] } \\
\hline $\begin{array}{l}\text { Modelo de negócio não é suficiente para } \\
\text { vencer os novos desafios }\end{array}$ & $\begin{array}{l}\text { Ciclos de desenvolvimento extremamente } \\
\text { reduzidos }\end{array}$ \\
\hline $\begin{array}{l}\text { Altas expectativas quanto às novas } \\
\text { tecnologias sendo implementadas }\end{array}$ & $\begin{array}{l}\text { Soluções tecnológicas tornam-se obsoletas } \\
\text { muito rapidamente }\end{array}$ \\
\hline $\begin{array}{l}\text { Falta de uma visão compartilhada da gerência } \\
\text { sobre como a empresa irá operar }\end{array}$ & $\begin{array}{l}\text { Escassez de pessoal com experiência prática } \\
\text { em novas tecnologias }\end{array}$ \\
\hline Muitas mudanças freqüentes e prematuras & $\begin{array}{l}\text { Equipes com perfis multidisciplinares } \\
\text { trabalhando físicamente separadas }\end{array}$ \\
\hline $\begin{array}{l}\text { Novas soluções introduzidas desestruturam os os } \\
\text { processos atuais }\end{array}$ & $\begin{array}{l}\text { Os requisitos podem ser comparados a um } \\
\text { alvo em movimento }\end{array}$ \\
\hline $\begin{array}{l}\text { Falta de um modelo de desenvolvimento de } \\
\text { comércio eletrônico }\end{array}$ & $\begin{array}{l}\text { Acesso de milhões de usuários com } \\
\text { expectativas e conhecimentos variados }\end{array}$ \\
\hline & Exposição aos melhores hackers do globo \\
\hline
\end{tabular}




\subsection{Problemas Levantados em Empresas Brasileiras}

O universo de empresas entrevistadas até o momento é constituído de: uma empresa incubada que está em fase de desenvolvimento de um pacote (suite) para comércio eletrônico; duas empresas que foram criadas para atender o mercado de telecomunicações e dadas as oportunidades de negócios começaram a atuar no mercado de aplicações para comércio eletrônico; e pelo braço "dotcom" de uma organização constituída de oito empresas atuando em diversos nichos do mercado de informática. Todas elas, com exceção da primeira, atuam em outros mercados além daqueles onde estão localizadas as matrizes. As entrevistas foram realizadas durante os meses de Março e Abril de 2002. Os perfis das empresas encontram-se na Tabela 2 e os problemas identificados durante as entrevistas na Figura 2.

Tabela 2: Perfil das empresas entrevistadas.

\begin{tabular}{|c|c|c|c|c|c|c|c|c|}
\hline \multirow[b]{2}{*}{ Pseudônimo } & \multirow[b]{2}{*}{$\mathbf{U F}$} & \multirow[b]{2}{*}{$\begin{array}{l}\text { Idade } \\
\text { (meses) }\end{array}$} & \multirow[b]{2}{*}{ Funcionários } & \multicolumn{3}{|c|}{ Projetos } & \multirow{2}{*}{$\begin{array}{l}\text { Tam. } \\
\text { Médio } \\
\text { das } \\
\text { Equipes }\end{array}$} & \multirow{2}{*}{\begin{tabular}{|l|} 
Duração \\
Média dos \\
Projetos \\
(meses)
\end{tabular}} \\
\hline & & & & B2C & B2B & Suite & & \\
\hline e-Alfa & SP & 4 & 3 & 0 & 0 & 1 & 3 & 0,0 \\
\hline e-Beta & $\mathrm{MG}$ & 48 & 25 & 2 & 0 & 0 & 4 & 5,5 \\
\hline e-Gama & SP & 80 & 110 & 2 & 2 & 0 & 11 & 8,0 \\
\hline e-Delta & SP & 44 & 30 & 2 & 0 & 0 & 4 & 6 \\
\hline
\end{tabular}

\begin{tabular}{|c|c|}
\hline GR & Gerência de Requisitos \\
\hline & Análise de Requisitos \\
\hline & Controle de Alterações \\
\hline EP & Engenharia de Produto \\
\hline & Documentação \\
\hline & Integração com Sistemas Legados \\
\hline & Testes \\
\hline GP & Gerência de Projeto \\
\hline & Acompanhamento de Projeto \\
\hline & Atrasos em Cronogramas \\
\hline & Garantia (alocação de tempo e recurso) \\
\hline$\overline{G C}$ & Gerência de Configuração \\
\hline & Versão \\
\hline PO & Processo da Organização \\
\hline & Institucionalização de Processos \\
\hline GT & Gerência de Tecnologia \\
\hline & Instrumentação \\
\hline
\end{tabular}

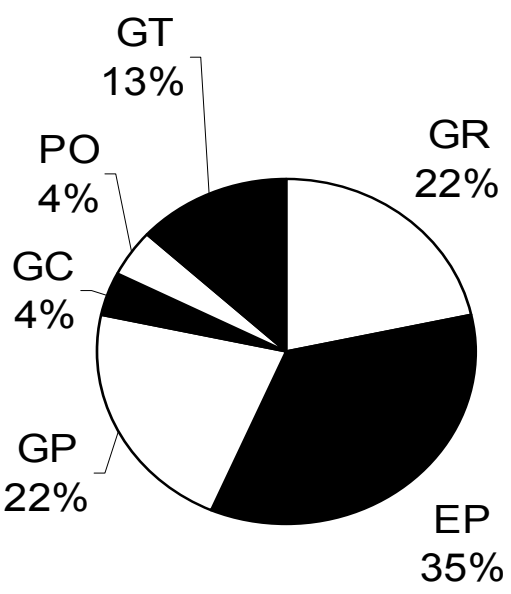

Figura 2: Distribuição de problemas levantados durante as entrevistas.

Como já foi dito anteriormente, o número de empresas entrevistadas é pequeno. Novas entrevistas serão realizadas para ampliar essa amostragem. Contudo, acredita-se que, baseado- 
se na literatura, a tendência ou distribuição de problemas, mostrada no quadro acima, se confirme.

\subsection{Problemas Levantados na Literatura}

Os problemas mostrados na Figura 3 foram levantados, em sete empresas, pelos autores das seguintes publicações: [2], [6], [8] e [17]. Os problemas ocorreram: em empresas de informática que desenvolvem aplicações para comércio eletrônico; empresas que desenvolvem produtos para apoiar desenvolvedores; e de empresas que não atuam no mercado de informática, mas que desenvolveram aplicações de comércio eletrônico com o objetivo de alavancar seus negócios.

\begin{tabular}{|ll|}
\hline GR & Gerência de Requisitos \\
\hline & Análise de Requisitos \\
& Incerteza dos Requisitos \\
& Absorção de Novas Funcionalidades pelo Usuário \\
& Priorização de Requisições \\
& Alteração de Requisitos \\
\hline EP & Engenharia de Produto de Software \\
\hline & Teste \\
& Processo de Criação de Web sites \\
\hline GP & Gerência de Projeto \\
\hline & Acompanhamento de Problemas \\
& Planejamento de Projeto \\
\hline GC & Gerência de Configuração \\
\hline & Versão \\
\hline PO & Processo da Organização \\
\hline & Práticas Inadequadas para Equipes Grandes \\
\hline RP & Revisão por Pares \\
\hline & Revisão por Pares \\
\hline
\end{tabular}

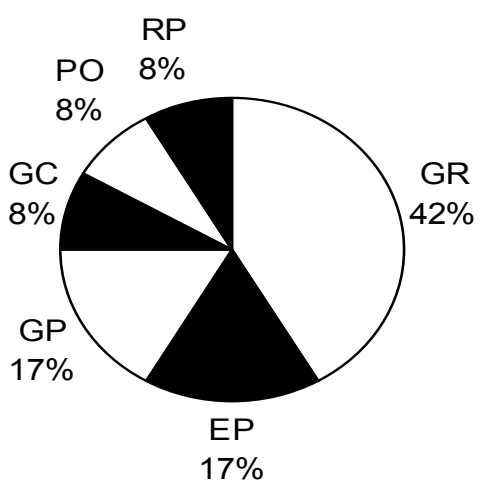

Figura 3: Distribuição dos problemas levantados na literatura.

\subsection{Análise dos Dados}

Uma análise preliminar dos dados coletados mostra que a natureza do desenvolvimento de software para comércio eletrônico é diferente do software convencional. O software para comércio eletrônico é bastante influenciado pelo mercado e pelas questões de negócios da empresa; os requisitos quase sempre não são ou não podem ser completamente definidos quando se realiza seu levantamento; o planejamento e acompanhamento de projetos são bastante dificultados pelo ambiente de incertezas; as metodologias, quando se tem alguma, são personalizadas; a composição da equipe é multidisciplinar e distribuída; e verifica-se uma grande dependência de ferramentas e tecnologia. Existe, ainda, uma grande preocupação com o "como fazer" e não com o "deve ser feito".

Além disso, em virtude dos curtos ciclos de desenvolvimento a qualidade quase sempre é negociada ou deixada em segundo plano. Em levantamentos feitos em [6] e [5], em um total de dezenove empresas norte-americanas de diversos setores que desenvolveram 
aplicações de comércio eletrônico, apenas três estavam ativamente implementando processos de melhoria de qualidade de software e somente em uma das empresas o processo era baseado no CMM. Nota-se, também, que os problemas levantados em diversas fontes da literatura e nas entrevistas têm um perfil bastante semelhante.

\section{Conclusão}

Os projetos de comércio eletrônico, mesmo em um ambiente de alta pressão, necessitam de métodos ou processos bem estruturados para obterem êxito. Os processos devem ser maduros, leves, ágeis e ter a qualidade de software como parte dos próprios processos. Dados preliminares coletados neste projeto indicam que os modelos mais consagrados não atendem bem a esses requisitos. Portanto, necessitam de ajustes para se adaptarem de forma eficiente à natureza crítica do ambiente Web e para elevar o nível de qualidade das implementações de comércio eletrônico sem, no entanto, se tornarem um gargalo no processo. No próximo passo do projeto, serão determinadas a aplicabilidade dos elementos dos modelos na solução dos problemas levantados e a eventual necessidade de adaptação.

\section{Referências Bibliográficas.}

[1] CÔRTES, M. , CHIOSSI, T. Modelos de Qualidade de Software. São Paulo: Unicamp, 2001. (Títulos em Engenharia de Software, 2.). ISBN: 852680551-7

[2] CUSUMANO, M.A., YOFFIE, D.B. Software Development on Internet Time. IEEE Computer, v. 32, n. 10, p. 60-69, Oct. 1999.

[3] FLORENZANO, V. Depois da Tempestade. InformationWeek Brasil: Especial e-Business, São Paulo:ITMidia, v.3, n.40, p. 9-17, fev. 2001.

[4] GÓIS, S.M. Algumas Considerações Sobre Comércio Eletrônico. Conjuntura \& Planejamento, Salvador:SEI/CPE, n.71, p. 25-26, abr. 2000.

[5] LEVINE L. et.al. Quality Software Development @Internet Speed, Briefing and Discussion of Findings. (Private Communication).

[6] LEVINE, L., BASKERVILLE, R., PRIES-HEJE, J. et al. How Internet Software Companies Negotiate Quality. IEEE Computer, v. 34, n. 5, p. 51-57, May. 2001.

[7] LEVINE, L., PRIES-HEJE, J. Process Improvement @ Internet Speed. In: Software Engineering Process Group (SEPG) Conference, Mar. 2001.

[8] MACCORMACK, A. Product-development practices that work: How Internet companies build software. Mit Sloan Management Review, v. 42, n. 2, p. 75-84, Winter 2001.

[9] PADUAN, R. Como a Internet está Transformando (de verdade) a Vida das Empresas. Exame Negócios, São Paulo:Abril, v.35, n.12, p. 46-58, jun. 2001.

[10] PAULK, M. C. "Internet Speed Processes" From a CMM Perspective. In: Eletronic Software Engineering Process Group(E-SEPG), Jun. 2001. Obtido via Internet: http://www.sei.cmu.edu/cmm/slides/is-cmm.pdf. 
[11] PAULK, M. et al. Capability Maturity Model for Software, Version 1.1. Software Engineering Institute, Carnegie Mellon University, CMU/SEI-93-TR-24, ESC-TR-93177, Feb. 1993. Obtido via Internet: http://www.sei.cmu.edu/pub/documents/93.reports/pdf/tr24.93.pdf.

[12] SHRUM, S. Choosing a CMMI Model Representation. CrossTalk, v. 13, n. 7, Jul 2000, pp.6-7.Obtido via Internet: http://www.stsc.hill.af.mil/crosstalk/2000/jul/jul00.pdf.

[13] SPRIGGS, J.E. CMMI for Systems Engineering/Software Engineering, Version 1.02 Staged Representation. Software Engineering Institute, Carnegie Mellon University, CMU/SEI-2000-TR-018, ESC-TR-2000-018, December 2000. Obtido via Internet: http://www.sei.cmu.edu/pub/documents/00.reports/pdf/00tr018.pdf.

[14] STRIGEL, W. Is Web Development a New Creature ?. E-ssential Newsletter, n.13, 23 Feb. 2000. Obtido via Internet: http://www.spc.ca/essentials/feb2300.htm.

[15] VIEIRA, E. Os donos do e-Commerce. Info Exame, São Paulo:Abril, ano 17, n.194,p. 6469, Mai. 2002.

[16] VU, J.D. The e-Commerce Capability Development Model: A Framework to Implement eCommerce Sucessfully. In: Software Engineering Process Group (SEPG) Conference, Fev. 2002. Obtido via Internet: http://itsqc.srv.cs.cmu.edu/eccm/SEI_SEPG_Tutorial_2002.pdf.

[17] WIEGERS, K. E. Software Process Improvement in Web Time. IEEE Software, v. 16, no. 4, pp. 78-86, Jul/Aug. 1999. Obtido via Internet: http://www.processimpact.com/articles/webtime.html.

[18] YOURDON, E.The Emergence of "Light" Development Methodologies. E-ssentials Newsletter , n. 30, 18 Oct. 2000. Obtido via Internet: http://www.spc.ca/essentials/oct1800.htm. 